\title{
Increased proliferation of middle to distal colonic cells during colorectal carcinogenesis in experimental murine ulcerative colitis
}

\author{
TAKUYA INOUE ${ }^{1}$, MITSUYUKI MURANO ${ }^{1}$, TAKANORI KURAMOTO ${ }^{1}$, KUMI ISHIDA $^{1}$, KEN KAWAKAMI $^{1}$, \\ YOSUKE ABE $^{1}$, EIJIRO MORITA ${ }^{1}$, NAOKO MURANO ${ }^{1}$, KEN TOSHINA $^{1}$, TAKASHI NISHIKAWA ${ }^{1}$, \\ KENTARO MAEMURA ${ }^{2}$, CHIKAO SHIMAMOTO ${ }^{1}$, ICHIRO HIRATA ${ }^{3}$, \\ KEN-ICHI KATSU ${ }^{1}$ and KAZUHIDE HIGUCHI ${ }^{1}$
}

\begin{abstract}
${ }^{1}$ Second Department of Internal Medicine, ${ }^{2}$ Department of Anatomy, Osaka Medical College, Osaka 569-8686; ${ }^{3}$ Department of Internal Medicine, Fujita Health University, 1-98 Dengakugakubo, Kutsukake-cho, Toyoake, Aichi 470-1192, Japan
\end{abstract}

Received May 14, 2007; Accepted September 3, 2007

\begin{abstract}
Patients with ulcerative colitis (UC) exhibit an increased risk for the development of cancer of the colon and rectum. This association is widely attributed to colonic inflammation. However, the severity of colonic inflammation necessary for the development of dysplasia and/or cancer remains unknown. In this study, we investigated the pattern of cell proliferation in colorectal carcinogenesis in an experimental murine model of UC. Chronic colitis was induced by administration of four cycles of dextran sulfate sodium (DSS) (each cycle: $5 \%$ or $2 \%$ DSS for 7 days and then distilled water for 14 days). Mice were sacrificed after every cycle and at 120 days following the completion of the fourth cycle. Colonic cell proliferation was immunohistochemically evaluated using the thymidine analogue bromodeoxyuridine and the labeling index (LI) was determined. The incidence of dysplasia and/or cancer was $28 \%, 6.7 \%$, and $0 \%$ in the $5 \%$ DSS, $2 \%$ DSS, and normal control groups respectively. All gross lesions were present in the middle to distal colon. Disease activity index and total LI after four cycles of DSS were significantly higher in the $5 \%$ DSS group compared to the $2 \%$ DSS group. In the $5 \%$ DSS group, the LI was significantly higher in the middle colon than in the proximal colon. Simple repeated administration of the non-genotoxic colon carcinogen DSS induced dysplasia and/or cancer. In addition, we have demonstrated the presence of regional differences in proliferation pattern between the middle and the proximal colon during carcinogenesis in experimental murine UC. These findings may provide insight into the development of colorectal cancer in humans with long-standing UC.
\end{abstract}

Correspondence to: Dr Mitsuyuki Murano, Second Department of Internal Medicine, Osaka Medical College, 2-7 Daigakumachi, Takatsuki city, Osaka 569-8686, Japan

E-mail: in2068@poh.osaka-med.ac.jp

Key words: dextran sulfate sodium, ulcerative colitis, dysplasia, cancer, proliferation

\section{Introduction}

Patients with ulcerative colitis (UC) exhibit an increased risk for the development of cancer of the colon and rectum with the risk of colorectal cancer increasing as the extent of colon affected and duration of disease increase. Indeed, the incidence of colorectal cancer in patients with long-standing UC is higher than that of sporadic colorectal cancer (1). Although this association is widely attributed to colonic inflammation, it is unknown whether the degree of inflammation relates to the development of dysplasia and/or cancer. Thus, experimental models of colitis that develop dysplasia and cancer similar to human UC are needed to investigate the dysplasia-cancer sequence and, although there are many animal models of inflammatory bowel disease (IBD), few are applicable to such study. Moreover, in most of these animal models, dysplasia and cancer are induced by pretreatment with genotoxic colon carcinogens such as azoxymethane (AOM) (2-4) or dimethylhydrazine $(\mathrm{DMH})(5,6)$ followed by dextran sulfate sodium (DSS) administration. These studies suggest that chronic or repeated mucosal inflammation induced by administration of DSS may result in the rapid development of colorectal cancer. On the other hand, in the absence of genotoxic colon carcinogens, the long-term treatment of mice with the nongenotoxic compound DSS results in dysplasia and cancer which has clinical and histopathological similarities to human UC (7-9).

Although experimental chronic colitis and colorectal cancer induced in rodents by administration of DSS has been extensively investigated, the mechanisms by which DSS induces both colonic inflammation and cancer are still unknown. For example, it is unclear whether dysplastic or proliferative changes occur first. However, abnormal proliferation is present in the normal-seeming mucosa of human UC (10). Therefore, abnormal epithelial cell proliferation is implicated in chronic colitis associated with subsequent carcinogenesis (11). Hyperproliferation of colonic epithelial cells was observed in tritiated thymidine-labeled specimens from carcinogen-treated mice (12). However, there have been no reports on the pattern of cell proliferation during carcinogenesis in experimental murine UC induced by simple repeated DSS administration. 
Bromodeoxyuridine (BrdU) is a thymidine analogue which becomes incorporated into DNA during the S-phase of the cell cycle and can be clearly detected using a monoclonal antibody (13). Several studies have demonstrated that the labeling index (LI) estimated by BrdU immunohistochemistry is equivalent to that obtained by thymidine autoradiography (14) and BrdU labeling has been considered to accurately represent the level of proliferative activity (15).

In the present study, we established a murine model of long-standing UC induced by simple repeated administration of the non-genotoxic colon carcinogen DSS. This resulted in the induction of dysplasia and/or cancer similar to UCassociated neoplasia although the number of lesions was relatively small. We then investigated the influence of the severity of colonic inflammation on colorectal carcinogenesis and regional differences in cell proliferation that might be associated with a higher incidence of tumors.

\section{Materials and methods}

Animals. Seven-week-old female BALB/c mice (CELA Japan, Tokyo, Japan) weighing 20-25 g were used in this study. The animals were maintained in an animal colony with controlled temperature $\left(23^{\circ} \mathrm{C}\right)$ and light $(12 / 12$-h light and dark cycle) at the Osaka Medical College (OMC), Osaka, Japan, and were permitted free access to standard mice chow pellets (MM-3, Funabashi, Chiba, Japan) and tap water.

Induction of experimental colitis. DSS (molecular weight 5000) was obtained from Meitou Sangyou (Osaka, Japan). Mice were given water containing $5 \%$ or $2 \%$ DSS instead of tap water on the indicated days.

Protocol for induction of colorectal tumors and experimental procedures. The experimental design is shown in Fig. 1. Chronic colitis was induced in mice by four cycles of oral administration of DSS with each cycle comprising DSS treatment for 7 days and distilled water for the following 14 days. Mice received intraperitoneal BrdU (B5002 Sigma Chemicals, St. Louis, MO, USA) at a dose of $40 \mathrm{mg} / \mathrm{kg}$ body weight $1 \mathrm{~h}$ before sacrifice. Cohorts of mice were sacrificed after every cycle to determine the labeling index, and at 120 days following the completion of the fourth cycle to evaluate the incidence of dysplasia and/or cancer. Mice were divided into three groups based on the amount of DSS they received: $5 \%$ DSS, $2 \%$ DSS and a normal control group.

Evaluation of the severity of clinical colitis. The disease activity index (DAI) was determined in all animals during the first administration cycle of DSS by scoring the extent of body weight loss, stool haemoccult reactivity or gross bleeding, and stool consistency in accordance with the method described by Murthy et al (16). This method of scoring is a comprehensive functional measure and correlates well with the degree of inflammation. The individuals who examined the mice and determined the DAI scores were blinded as to the experimental group to which the animal belonged.

Pathologic examination. After sacrifice, the entire colorectum from the colocecal junction to the anal verge was excised and

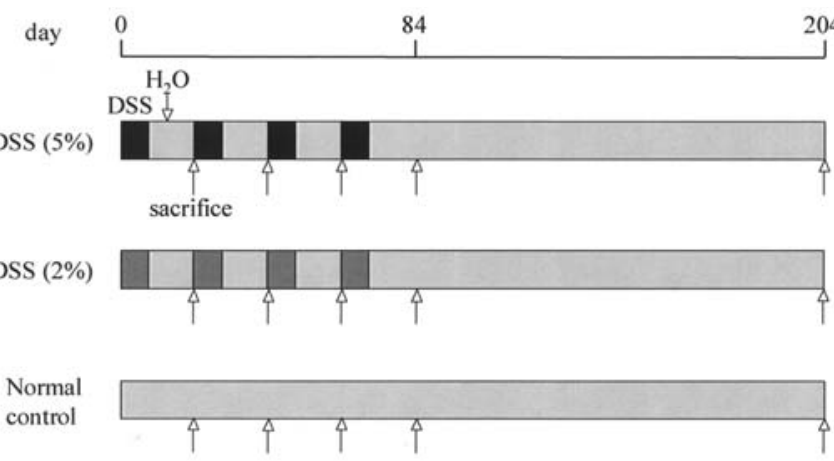

Figure 1. The protocol used for inducing colorectal tumors in association with ulcerative colitis in mice by intermittent administration of $5 \%$ or $2 \%$ dextran sulfate sodium (DSS).

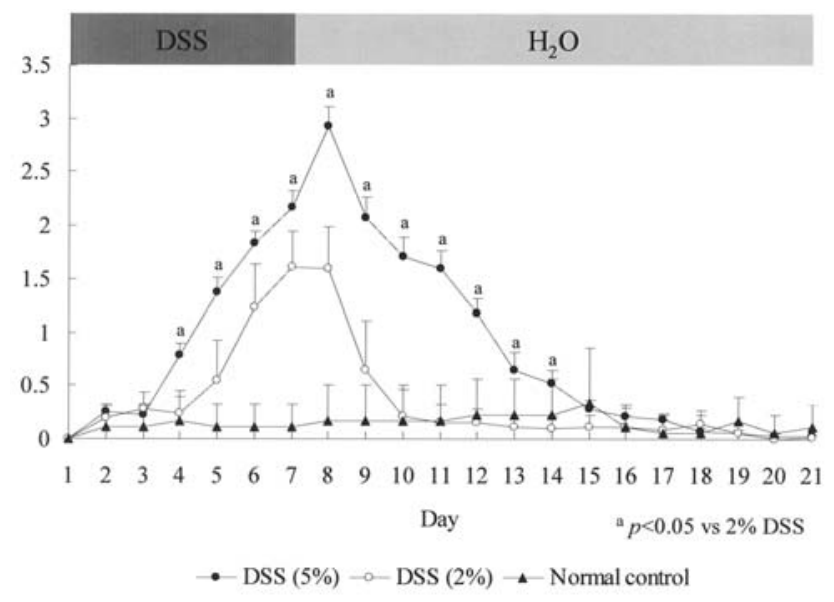

Figure 2. Time course of DAI score after the start of DSS administration.

rinsed in phosphate-buffered saline (PBS). The specimen was opened longitudinally, examined for gross lesions and all gross lesions were recorded. The colon was divided into three equal portions (proximal, middle and distal) and each segment was fixed in $10 \%$ formalin. Subsequently, three transverse sections of each segment were submitted for histological processing. All slides were stained with hematoxylin and eosin.

According to the histological criteria outlined by the Inflammatory Bowel Disease - Dysplasia Morphology Study Group (17), dysplasia was categorized on the basis of microscopic findings. This included architectural alteration beyond the degree of reparative changes in chronic colitis, which often resembled the glandular arrangement of adenomas, and cytologic abnormalities that consisted principally of cellular and nuclear pleomorphism, nuclear hyperchromatism, loss of nuclear polarity and marked stratification of nuclei. The cancer category included 'early invasive', which was defined as cancer cells invading the muscularis mucosa and/or the submucosa. The indefinite category consisted of 'cytologically atypical epithelium', which was recognized for the most part to be reparative and/or regenerative in nature.

BrdU immunohistochemistry. Expression of BrdU in the intestinal mucosa was assessed by the labeled streptavidin biotin method using an LSAB kit (Dako, Carpinteria, CA, 
Table I. Colorectal length and gross lesion position.

\begin{tabular}{lccc}
\hline & DSS (5\%) & DSS (2\%) & $\begin{array}{c}\text { Normal } \\
\text { control }\end{array}$ \\
\hline $\begin{array}{l}\text { Colon length (cm) } \\
\begin{array}{l}\text { Distance from anus of } \\
\text { gross lesions }(\mathrm{cm})\end{array}\end{array}$ & $2.7 \pm 1.2$ & 2.3 & $/$ \\
\hline
\end{tabular}

USA). The entire colon was divided into three equal portions (proximal, middle and distal) and each longitudinal segment was fixed in $10 \%$ formalin, embedded in paraffin wax and cut into tissue sections of $4 \mu \mathrm{m}$ thickness. Tissue sections were mounted on microscope slides, dewaxed in xylene (3x3 min) and dehydrated with $100 \%$ ethanol. After washing with PBS, sections were treated with $2 \mathrm{~mol} / \mathrm{l} \mathrm{HCL}$ for $30 \mathrm{~min}$ and then treated twice with $0.1 \mathrm{~mol} / 1$ borate buffer $(\mathrm{pH} 8.5)$ for $5 \mathrm{~min}$. After washing with PBS, endogenous peroxidase activity was blocked using $0.3 \%$ hydrogen peroxidase in $10 \%$ methanol for $30 \mathrm{~min}$ and blocking reagent was added for $15 \mathrm{~min}$. Sections were incubated at $4^{\circ} \mathrm{C}$ overnight with the primary mouse monoclonal antibody ( 1 in 20 dilution in PBS) against BrdU (Dako). After washing with PBS, sections were incubated with a biotinylated anti-mouse immunoglobulin antibody (Dako) at room temperature for $30 \mathrm{~min}$. Sections were then washed in PBS and visualized using streptavidin-biotinhorseradish peroxidase (Dako) and 3, 3' diaminobenzidine (Dako). Finally, sections were counterstained with Meyer's hematoxylin solution, dehydrated and cover-slipped with permanent mounting medium for microscopic examination.

Evaluation of the labeling index (LI). Only complete wellorientated longitudinally sectioned crypts displaying the lumen at the top and the muscularis mucosa at the base were used for analysis. Labeled cells were defined as epithelial cells whose nuclei were stained brown at a magnification of $\mathrm{x} 400$. The LI for the whole crypt and for each compartment (proximal, middle and distal) was determined after counting the number of BrdU-labeled cells per 10 crypts and dividing the number of labeled cells by the total cell number and multiplying by 100 .

Statistical analysis. All results are expressed as mean \pm SD. Comparisons were performed using one-way ANOVA followed by Tukey-Kramer's test. Categorical data were analyzed by the chi-squared test. Statistical significance was defined as $\mathrm{P}<0.05$.

\section{Results}

Changes of DAI score. Most mice in the 5\% DSS group exhibited loose and haemoccult-positive stools 2 days after DSS administration (day 2). Clinical symptoms of colitis including bloody stool, diarrhea and loss of body weight progressed further until day 8 but then gradually disappeared during the subsequent 14-day period of drinking distilled water without DSS. Accordingly, the DAI score gradually
Table II. Incidence of dysplasia and/or cancer in mice with DSS-induced ulcerative colitis.

\begin{tabular}{lcccc}
\hline & $\begin{array}{c}\text { Negative } \\
\text { and/or } \\
\text { indefinite }\end{array}$ & Dysplasia & Cancer & $\begin{array}{c}\text { Dysplasia } \\
\text { and/or } \\
\text { cancer }\end{array}$ \\
\hline $\begin{array}{l}5 \% \text { DSS } \\
(\mathrm{n}=25)\end{array}$ & $18(72 \%)$ & $7(28 \%)$ & $5(20 \%)$ & $7(28 \%)$ \\
$\begin{array}{l}2 \% \text { DSS } \\
(\mathrm{n}=15)\end{array}$ & $14(93.3 \%)$ & $1(6.7 \%)$ & $0(0 \%)$ & $1(6.7 \%)$ \\
$\begin{array}{l}\text { Normal } \\
\text { control } \\
(\mathrm{n}=10)\end{array}$ & $10(100 \%)$ & $0(0 \%)$ & $0(0 \%)$ & $0(0 \%)$ \\
\hline
\end{tabular}

increased from day 2 to day 8 and usually reverted to normal by day 21 . Treatment of mice with $5 \%$ DSS significantly increased the DAI score from day 4 to 14 compared to the $2 \%$ DSS group (Fig. 2)

Colorectal length, incidence and distribution of dysplasia and/or cancer and tumor size. The colorectal length was significantly shortened compared to the normal control group after 120 days from the completion of the fourth cycle of DSS administration. The colorectal length in the 5\% DSS, $2 \%$ DSS and normal control groups was $8.2 \pm 0.9 \mathrm{~cm}, 9.1 \pm 0.6 \mathrm{~cm}$ and $12.1 \pm 0.6 \mathrm{~cm}$, respectively. All gross lesions had the shape of the sessile type and were observed in the middle or distal colon (Table I) whilst all mice with dysplasia and/or cancer had lesions limited to only one colon segment (Fig. 3). The size of gross lesions in the $5 \%$ and $2 \%$ DSS groups was $4480 \pm 1184 \mu \mathrm{m}$ and $2000 \mu \mathrm{m}$, respectively. The incidence of dysplasia and/or cancer is presented in Table II. The incidence of dysplasia and/or cancer in the $5 \%$ and $2 \%$ DSS group was $28.0 \%$ and $6.7 \%$, respectively.

BrdU labeling in the colorectum. The time course study of in vivo BrdU uptake is shown in Fig. 4. Although BrdU immunoreactive cells amounted to only $0.71 \pm 0.34 \%$ of total cells in the whole colon of normal control mice, the percentage of BrdU-positive cells after four cycles of DSS administration increased to $7.69 \pm 1.86 \%$ and $4.56 \pm 2.21 \%$ in the $5 \%$ DSS and $2 \%$ DSS groups respectively. The total LI was significantly higher in the 5\% DSS group than in the 2\% DSS group. Concerning regional differences, the LI was significantly greater in the middle colon $(8.5 \pm 1.65 \%)$ than in the proximal colon $(6.33 \pm 1.95 \%)$ in the $5 \%$ DSS group after four cycles of DSS administration (Table III).

\section{Discussion}

DSS is a synthetic sulfate polysaccharide composed of dextran with sulfate glucose and is known to induce colitis, and DSS-induced murine colitis has been used as a model of human UC $(5,8)$. The mechanisms of DSS-induced colitis are believed to involve macrophage dysfunction, altered luminal bacterial flora and toxic effects upon the colonic epithelium 
$\mathbf{A}$

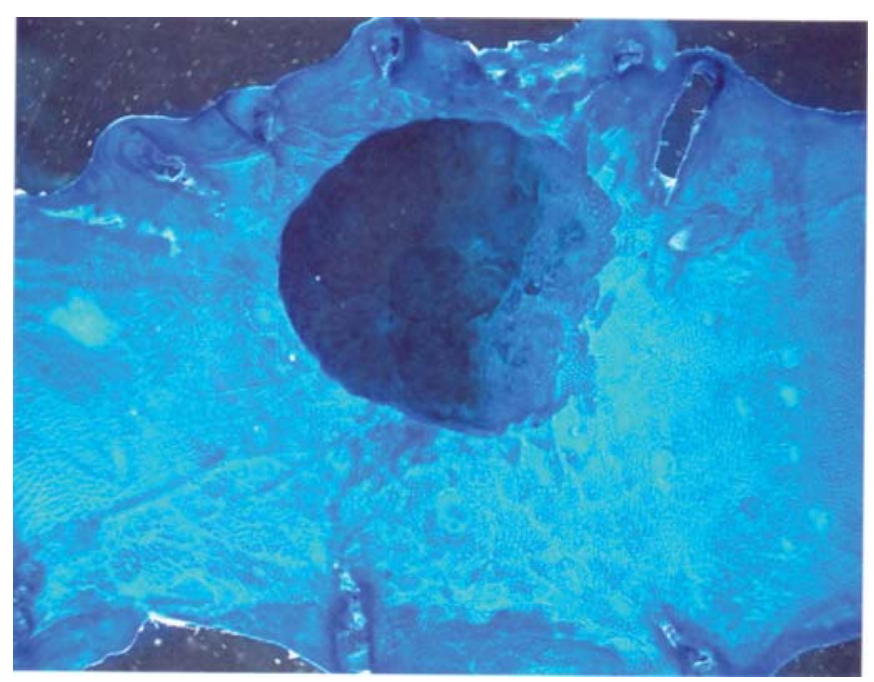

B

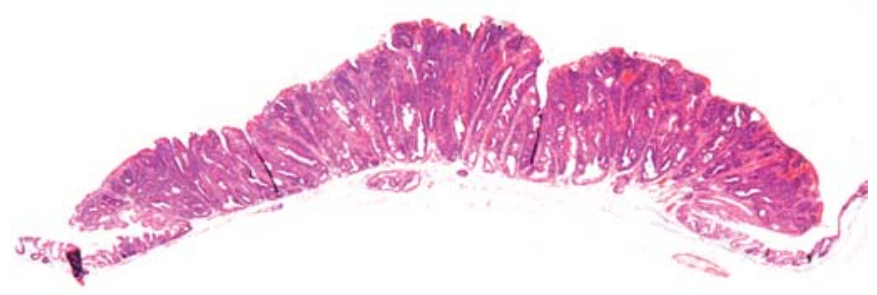

C

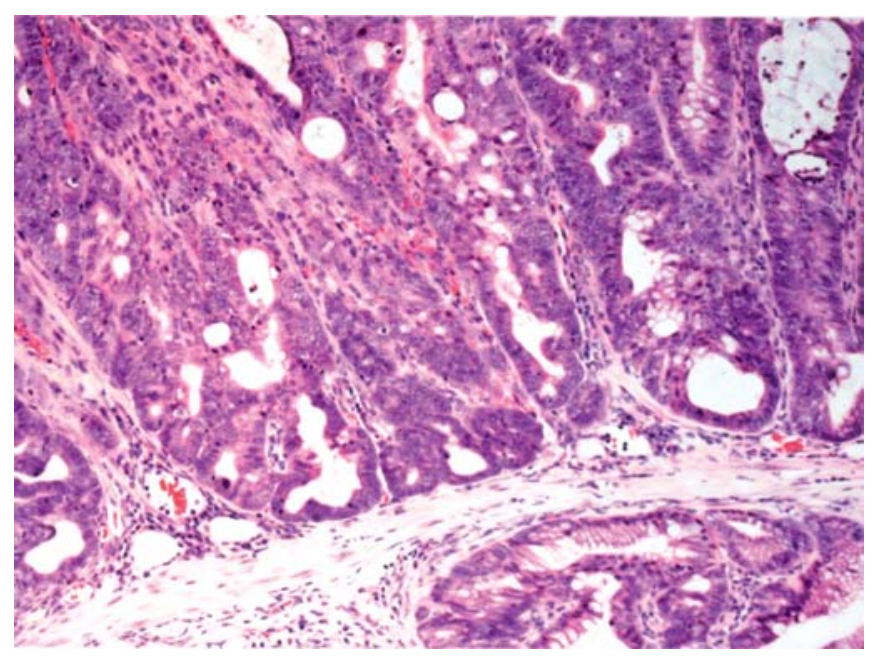

$(18,19)$. Colorectal cancer is one of the most serious complications of UC and the risk of UC-associated neoplasia increases as the extent and duration of the disease increase. Indeed, the incidence of colorectal cancer in patients with long-standing UC is higher than that of sporadic colorectal cancer (1). A few investigators have described the appearance of dysplasia and/or cancer in mice following simple repeated administration of the non-genotoxic colon carcinogen DSS and suggested that repeated mucosal erosion with necrosis
D

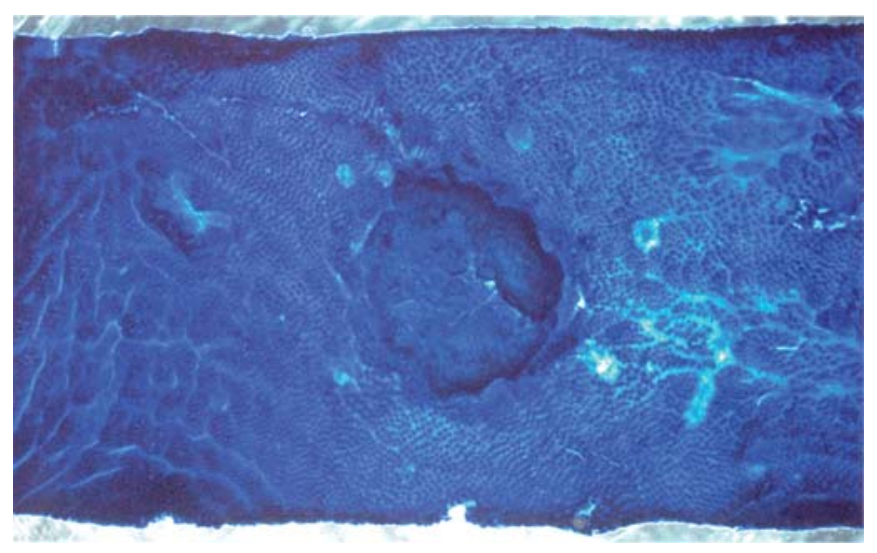

$\mathbf{E}$

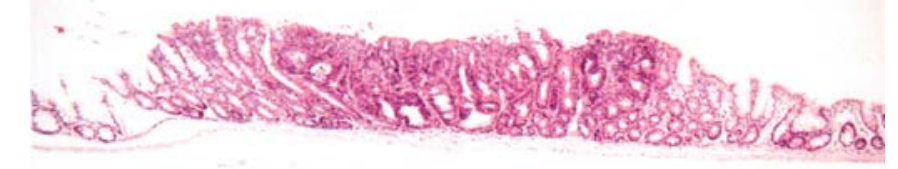

$\mathbf{F}$

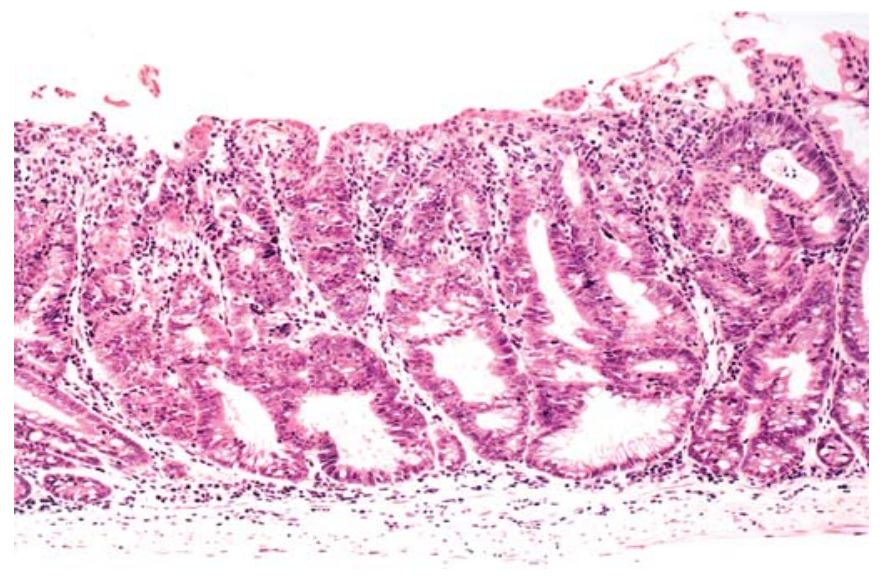

Figure 3. Neoplastic lesions in mice with DSS-induced ulcerative colitis. A, stereoscopic view. A $6.5-\mathrm{mm}$ sessile type is elevated on the colonic mucosa in the 5\% DSS group. B, whole mount of view. Tumor cells are invading into the submucosa. $\mathrm{C}$, the tumor was diagnosed as an invasive cancer. D, stereoscopic view. A 2.0-mm sessile type is elevated on the colonic mucosa in the $2 \%$ DSS group. E, whole mount of view. A slightly elevated lesion demonstrates cellular and structural atypia. F, the tumor was diagnosed as a dysplasia.

and regeneration of the colonic epithelium seems to increase the susceptibility of the mucosal epithelia for dysplasia and/or cancer development (7). In the present study, simple repeated administration of the non-genotoxic colon carcinogen DSS induced dysplasia and/or cancer in mice, though the neoplastic lesions were relatively few in number compared to those induced by genotoxic carcinogens such as AOM and DMH. Interestingly, in contrast to human UC, there were no mice that exhibited more than one neoplasm in this study. Using 


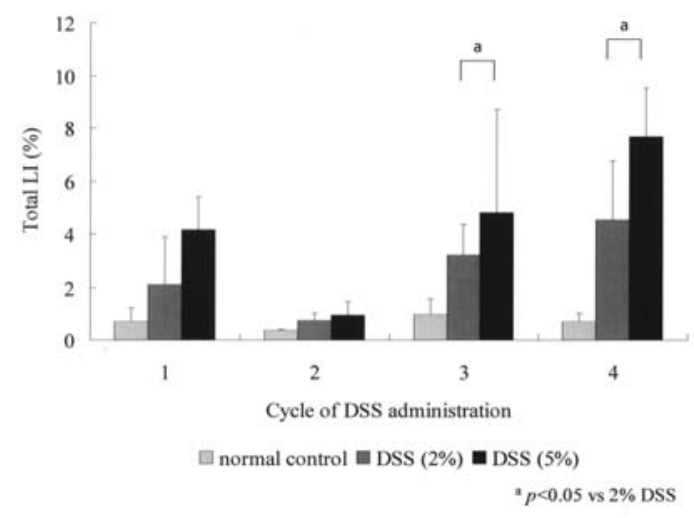

Figure 4. Comparison of cell proliferation during the time course study of four cycles of DSS administration.

Table III. Comparison of cell proliferation in the colon segments after four cycles of DSS administration.

\begin{tabular}{lcccc}
\hline & Proximal & Middle & Distal & Total \\
\hline $\begin{array}{l}5 \% \text { DSS } \\
(\mathrm{n}=4)\end{array}$ & $6.33 \pm 1.95$ & $8.5 \pm 1.65^{\mathrm{a}}$ & $8.23 \pm 0.46$ & $7.69 \pm 1.86$ \\
$\begin{array}{l}2 \% \text { DSS } \\
(\mathrm{n}=4)\end{array}$ & $3.92 \pm 1.61$ & $6.32 \pm 2.84$ & $3.43 \pm 0.89$ & $4.56 \pm 2.21$ \\
$\begin{array}{l}\text { Normal } \\
\text { control } \\
(\mathrm{n}=4)\end{array}$ & $0.41 \pm 0.24$ & $0.82 \pm 0.56$ & $0.76 \pm 0.6$ & $0.71 \pm 0.34$ \\
\hline
\end{tabular}

${ }^{\mathrm{a}} \mathrm{p}<0.05$ vs proximal.

the same murine model, Cooper et al also described that $87.5 \%$ of mice with dysplasia and/or cancer had lesions limited to only one colon segment with $33.3 \%$ of cancers being associated with dysplasia (7).

Although the underlying mechanisms of the frequent development of colonic dysplasia and/or cancer with chronic colonic inflammation are still unknown, Vetuschi et al examined epithelial cell proliferation and apoptosis of colonic mucosa in DSS-induced colitis in rats and reported that abnormal epithelial hyperproliferation could be involved in the high frequency of colorectal cancer (11). However, this study examined only the acute phase of DSS-induced colitis. Therefore we investigated epithelial proliferation at the end of every cycle when the DAI score usually reverted to normal, and showed persistent epithelial proliferation in this chronic colitis model. Shinozaki et al investigated the level of proliferation in apparently normal mucosa in human UC and reported that the presence of high background proliferative activity in the mucosa suggested that increased proliferative activity preceded dysplasia (10). Although it is still unknown whether levels of inflammation relate to the development of dysplasia and/or cancer $(7,20)$, these changes could be attributed to recurrent cycles of inflammation and regeneration of the colonic mucosa in UC $(11,21)$. Therefore in the present study, we examined groups with different DAI levels to investigate whether the levels of inflammation related to the development of dysplasia and/or cancer, and demonstrated that animals with a higher DAI had more dysplasia and/or cancer compared to those with lower DAI, though the differences were not statistically significant (Table II; $\mathrm{p}=0.1025$ ).

In the present study, we performed a time-course study of LI during four cycles of DSS administration and demonstrated that the LI gradually increased from the first to the fourth cycle and showed significant differences associated with different DAI levels. It seems likely that the necrosis and regeneration of colorectal mucosa facilitates persistent epithelial hyperproliferation $(8,22)$. Previous studies have shown that colonic epithelial cells of human UC exhibit activated nuclear factor- $\mathrm{kB}(\mathrm{NF}-\mathrm{\kappa B})$, which is an important regulator of proinflammatory cytokine production and inhibitor of apoptosis (23-25). This suggests the involvement of inflammatory cytokines in both inflammation-associated cell death and the activation of cellular protection (23). On the other hand, persistent cellular resistance to apoptosis due to chronic inflammation may be associated with a disruption in the critical balance between epithelial cell apoptosis and proliferation and lead to the subsequent development of dysplasia $(8,26,27)$.

Concerning the distribution of BrdU-labeled cells, we demonstrated significant differences between the proximal and middle colon where many gross lesions were observed. Ma et al also demonstrated significant regional differences in the distribution of BrdU-labeled cells in proximal and distal colon in DMH-treated rats (13). Therefore, we consider regional differences in cell proliferation to relate to the severity of inflammation and possibly be associated with a higher incidence of tumors in the middle to distal colon than in the proximal colon. Also, we suggest that the persistent background proliferative change that may precede mucosal dysplasia plays an important role in colorectal carcinogenesis in chronic colitis. In cancer tissues, the proportion of proliferating cells increases whilst the proportion of apoptotic cells decreases such that the ratio between apoptosis and proliferation falls (28). However, nitrosative stress can both prevent and induce apoptosis and it is still unknown whether the level of inflammation is a key regulator of apoptosis in this biological context $(29,30)$.

In conclusion, the present data demonstrate that simple repeated administration of the non-genotoxic colon carcinogen DSS induced dysplasia and/or cancer and that the high level of inflammation could be an important factor in the earlier stage of initiation of dysplasia. Although further studies are needed to clarify the relationship between continued inflammation and apoptosis, a persistent abnormal level of background cell proliferation of the mucosa together with regional differences in cell proliferation may play an important role in the development of dysplasia and/or cancer in this murine model of colitis. These findings may also provide insight into the development of colorectal cancer in humans with long-standing ulcerative colitis.

\section{Acknowledgements}

This study was partially supported by a grant from the Osaka Medical Research Foundation for Incurable Disease. 


\section{References}

1. Ekbom A, Helmick C, Zack M and Adami HO: Ulcerative colitis and colorectal cancer. A population-based study. N Eng J Med 323: 1228-1233, 1990

2. Okayasu I, Ohkusa T, Kajiura K, Kanno J and Sakamoto S: Promotion of colorectal neoplasia in experimental murine ulcerative colitis. Gut 39: 87-92, 1996.

3. Tanaka T, Kohno H, Suzuki R, Yamado Y, Sugie S and Mori H: A novel inflammation-related mouse colon carcinogenesis model induced by azoxymethane and dextran sodium sulfate. Cancer Sci 94: 965-973, 2003.

4. Suzuki R, Kohno H, Sugie S and Tanaka T: Sequential observations on the occurrence of preneoplastic and neoplastic lesions in mouse colon treated with azoxymethane and dextran sodium sulfate. Cancer Sci 95: 721-727, 2004.

5. Onose J, Imai T, Hasumura M, Ueda M and Hirose M: Rapid induction of colorectal tumors in rats initiated with 1,2dimethylhydrazine followed by dextran sodium sulfate treatment. Cancer Lett 198: 145-152, 2003.

6. Wang JG, Wang DF, Lv BJ and Si JM: A novel mouse model for colitis-associated colon carcinogenesis induced by 1,2 dimethylhydrazine and dextran sulfate sodium. World J Gastroenterol 10: 2958-2962, 2004.

7. Cooper HS, Murthy S, Kido K, Yoshitake H and Flanigan A: Dysplasia and cancer in the dextran sulfate sodium mouse colitis model. Relevance to colitis-associated neoplasia in the human: a study of histopathology, ß-catenin and p53 expression and the role of inflammation. Carcinogenesis 21: 757-768, 2000.

8. Okayasu I, Yamada M, Mikami T, Yoshida T, Kanno J and Ohkusa T: Dysplasia and carcinoma development in a repeated dextran sulfate sodium-induced colitis model. J Gastroenterol Hepatol 17: 1078-1083, 2002.

9. Korenaga D, Takesue F, Kido K, Yasuda M, Inutsuka S, Honda $\mathrm{M}$ and Nagahama S: Impaired antioxidant defense system of colonic tissue and cancer development in dextran sulfate sodium-induced colitis in mice. J Surg Res 102: 144-149, 2002.

10. Shinozaki M, Watanabe T, Kubota Y, Sawada T, Nagawa H and Muto T: High proliferative activity is associated with dysplasia in ulcerative colitis. Dis Colon Rectum 43: S34-S39, 2000.

11. Vetuschi A, Latella G, Sferra R, Caprilli R and Gaudio E: Increased proliferation and apoptosis of colonic epithelial cells in dextran sulfate sodium-induced colitis in rats. Dig Dis Sci 47: 1447-1457, 2002.

12. Chang WW: The mode of growth and compartmentalization of neoplastic glands during experimental colon carcinogenesis. Am J Pathol 124: 420-426, 1986.

13. Ma QY, Williamson KE and Rowlands BJ: Variability of cell proliferation in the proximal and distal colon of normal rats and rats with dimethylhydrazine induced carcinogenesis. World J Gastroenterol 8: 847-852, 2002.

14. Qin Y and Willems G: Comparison of the classical autoradiographic and the immunohistochemical methods with BrdU for measuring proliferation parameters in colon cancer. Anticancer Res 13: 731-735, 1993.
15. Mitamura T, Sakamoto S, Sassa S, Suzuki S, Kudo H and Okayasu I: The more an ulcerative colitis is repeated, the more the risk of colorectal carcinogenesis is increased in mice. Anticancer Res 22: 3955-3961, 2002.

16. Murthy SN, Cooper HS, Shim H, Shah RS, Ibrahim SA and Sedergran DJ: Treatment of dextran sodium-induced murine colitis by intracolonic cyclosporine. Dig Dis Sci 38: 1722-1734, 1993.

17. Riddell RH, Goldman H, Ransohoff DF, Appelman HD, Fenoglio CM, Haggitt RC, Ahren C, Correa P, Hamilton SR, Morson BC, Sommers SC and Yardley JH: Dysplasia in inflammatory bowel disease: standardized classification with provisional clinical applications. Hum Pathol 14: 931-968, 1983.

18. Orii S, Yamaguchi T, Anzai H, Saito S, Chiba T and Suzuki K: Chemoprevention for colorectal tumorigenesis associated with chronic colitis in mice via apoptosis. J Exp Clin Cancer Res 22: 41-46, 2003

19. Kim HS and Berstad A: Experimental colitis in animal models. Scand J Gastroenterol 27: 529-537, 1992.

20. Pinczowski D, Ekbom A, Baron J, Yuen J and Adami HO: Risk factors for colorectal cancer in patients with ulcerative colitis: a case-control study. Gastroenterology 107: 117-120, 1994.

21. Okayasu I, Hatakeyama S, Yamada M, Ohkusa T, Inagaki Y and Nakaya R: A novel method in the induction of reliable experimental acute and chronic ulcerative colitis mice. Gastroenterology 98: 694-701, 1990.

22. Serafini EP, Kirk AP and Chambers TJ: Rate and pattern of epithelial cell proliferation in ulcerative colitis. Gut 22: 648-652, 1981.

23. Iimura M, Nakamura T, Shinozaki S, Iizuka B, Inoue Y, Suzuki S and Hayashi N: Bax is downregulated in inflamed colonic mucosa of ulcerative colitis. Gut 47: 228-235, 2000.

24. Rogler G, Brand K, Vogl D, Page S, Hofmeister R, Andus T, Knuechel R, Baeuerle PA, Scholmerich J and Gross V: Nuclear factor kappa B is activated in macrophages and epithelial cells of inflamed intestinal mucosa. Gastroenterology 115: 357-369, 1998.

25. Barinaga M: Life-death balance within the cell. Science 274 724,1996 .

26. Mannick JB, Miao XQ and Stamler JS: Nitric oxide inhibits Fas-induced apoptosis. J Biol Chem 272: 24125-24128, 1997.

27. Wenzel U, Kuntz S, Sousa UJ and Daniel H: Nitric oxide suppresses apoptosis in human colon cancer cells by scavenging mitochondrial superoxide anions. Int J Cancer 106: 666-675, 2003

28. Sun BC, Zhao XL, Zhang SW, Liu YX, Wang L and Wang X Sulindac induces apoptosis and protects against colon carcinoma in mice. World J Gastroenterol 11: 2822-2826, 2005.

29. Kim KM, Kim PK, Kwon YG, Bai SK, Nam WD and Kim YM Regulation of apoptosis by nitrosative stress. J Biochem Mol Biol 35: 127-133, 2002.

30. Liu Q, Chan ST and Mahendran R: Nitric oxide induces cyclooxygenase expression and inhibits cell growth in colon cancer cell lines. Carcinogenesis 24: 637-642, 2003. 